\title{
Clinical Study Actual Accrual Number
}

National Cancer Institute

\section{Source}

National Cancer Institute. Clinical Study Actual Accrual Number. NCI Thesaurus. Code C70760.

A number of subjects that have been enrolled for the participation in a clinical study at a given point in time. A date for the actual accrual number must be specified. 